\title{
SIMPLE SOLUTIONS FOR APPROXIMATE TREE MATCHING PROBLEMS
}

\author{
Fabrizio Luccio Linda Pagli \\ Dipartimento di Informatica, Università di Pisa \\ Corso Italia 40, 56100 Pisa, Italy
}

\section{Introduction}

Tree isomorphism and matching is widely studied. Aside from its mathematical interest, the problem has a number of important motivations in the theory of programming [4] and in molecular biology [9]. Three recent papers in this field are particularly innovative by a computational point of view. Given a pattern tree $\mathrm{P}$ and a text tree T, Mäkinen [7] shows how to determine all the exact occurrences of $P$ as subtree of $T$, in time $\mathrm{O}(|\mathrm{P}|+|\mathrm{T}|)$, using a proper string representation for trees, and applying a fast string matching algorithms on such a string. In [5] Kosaraju solves a generalization of the same problem, generally called tree pattern matching, where the leaves of $\mathrm{P}$ may match with subtrees of $\mathrm{T}$ (instead of single nodes). The time required by the algorithm of $[5]$ is $\mathrm{O}(|\mathrm{T}| \mathrm{PP} \mid 0.75$ polylog|PI) against the $\mathrm{O}(|\mathrm{T}||\mathrm{P}|)$ of the naive bound. In fact the algorithm of [5] solves the more general problem of determining whether $P$ is isomorphic to a subtree $T^{\prime}$ of $T$, where an arbitrary number of subtrees may be cancelled from $T^{\prime}$. The result of [5] has been improved in [1], to $O\left(n \sqrt{ } V_{m}\right.$ polylog $\left.(m)\right)$. Zhang and Shasha [9] measure the edit distance between ordered trees (weighted number of insert, delete and modify operations to trasform one tree into another), and consider several approximate tree matching problems based on this distance, or other weighted distances derived from subtree removal or pruning. Due to the intrinsic difficulty of their problems, all their algorithms run in more than linear time. Among the classical contributions, Hoffmann and O'Donnell [4] propose several algorithms for the subtree replacement problem in an ordered ranked tree. Unless for special cases, the algorithms of [4] are at least quadratic in the size of the input.

In this paper we consider ordered $h$-ary trees, that is trees whose nodes have exactly $h$ sons; and arbitrary ordered trees. The former appear, for example, in biochemical structures such as glycogen [8]; the latter are encountered in term rewriting systems and in RNA comparisons. We define the subtree distance between two ordered $h$-trees $T_{1}, T_{2}$ as the number of subtrees to be inserted or deleted in $T_{1}$ to obtain $T_{2}$, and introduce an approximate tree matching problem (Problem 1), that consists of finding all the occurrences of $P$ in $T$, with bounded distance $\mathrm{k}$. On one side, this problem is an extension of the general problem solved in [5], where $\mathrm{P}$ may contain subtrees which are not present in $\mathrm{T}$ (note however the h-ary restriction on $\mathrm{P}$ and $\mathrm{T}$ ). On another side, it is an extension of the approximate string matching problem $[2,6]$ to h-ary trees. We give an algorithm to solve this problem in time $\mathrm{O}(\mathrm{h} / \mathrm{PI}+$ $\max (\mathrm{h}, \mathrm{k})|\mathrm{T}|)$.

For arbitrary ordered trees we solve an extension of the tree pattern matching problem. We define the leaf distance between two trees $T_{1}, T_{2}$ as the total number of subtrees to be inserted in $T_{1}$ in place of its leaves, or to be deleted from $T_{1}$, leaving leaves in their place, to obtain $T_{2}$. 
Our new problem (Problem 2) consists of determining all the occurrences of $P$ as a subtree of $\mathrm{T}$, with bounded distance $\mathrm{k}$. Note that, unlike in the tree pattern matching problem, $\mathrm{P}$ is not necessarily contained in $T$. The complexity of our algorithm is $\mathrm{O}(|\mathrm{P}|+\mathrm{k}|\mathrm{T}|)$. With an obvious restriction, the algorithm can be adapted to solve the tree pattern matching problem with the same complexity. If $\mathrm{k}$ is chosen independently of $\mathrm{IPI}$, as it is normally assumed in approximate string matching, our algorithm compares favourably with the previous ones.

Problems 1 and 2 are defined for unlabelled trees; the extension to labelled trees require minor modifications of the algorithms. If up to $\mathrm{k}_{1}$ label differences are allowed between the matching nodes of $\mathrm{P}$ and $\mathrm{T}$, the two problems are solved in time $\mathrm{O}\left(\mathrm{h}|\mathrm{P}|+\max \left(\mathrm{h}, \mathrm{k}, \mathrm{k}_{1}\right)|\mathrm{T}|\right)$, and $\mathrm{O}\left(\mathrm{IPI}+\max \left(\mathrm{k}, \mathrm{k}_{1}\right) \mid \mathrm{Tl}\right)$, respectively.

\section{Matching h-ary trees}

Our study begins with a definition of tree distance. Let an ordered h-ary tree $\mathrm{T}$ be completed by the insertion of (h-1)|Tl+1 external nodes, in place of the empty sons of the original (internal) nodes. If $|\mathrm{T}|=0$ (i.e., $\mathrm{T}=\varnothing$ ) one external node is placed at the root. For a node $\mathrm{N} \in \mathrm{T}, \mathrm{N}$ internal or external, let $\mathrm{T}[\mathrm{N}]$ denote the subtree of $\mathrm{T}$ rooted at $\mathrm{N}$. We define the following operations on $\mathrm{T}$ :

1) for $\mathrm{N} \in \mathrm{T}, \mathrm{N}$ external, insert a new tree $\mathrm{T}_{1}$ in $\mathrm{T}$ as a subtree rooted at $\mathrm{N}$. This is denoted by: Ins $\left(\mathrm{T}_{1}, \mathrm{~T}[\mathrm{~N}]\right)$;

2) for $\mathrm{N} \in \mathrm{T}, \mathrm{N}$ internal, delete $\mathrm{T}[\mathrm{N}]$ from $\mathrm{T}$ (i.e., substitute $\mathrm{T}[\mathrm{N}]$ with an external node). This is denoted by: $\operatorname{Del}(\mathrm{T}[\mathrm{N}], \mathrm{T})$.

The transformation from $T_{1}$ to $T_{2}$, denoted by $T_{1} \alpha_{S} T_{2}$, is a sequence $S$ of insert or delete operations on $\mathrm{T}_{1}$. In this transformation we establish a correspondence between pairs of internal nodes $N_{1} \in T_{1}, N_{2} \in T_{2}$, denoted by $N_{1} \equiv N_{2}$. Let $R_{1}, R_{2}$ be the roots of $T_{1}, T_{2}$, respectively. The sequence $S$ is such that:

1) if $T_{1}=T_{2}=\varnothing$ then $S=\varnothing$;

2) if $T_{1}=\varnothing\left(R_{1}\right.$ is an external node) and $T_{2} \neq \varnothing$, then $S=\operatorname{Ins}\left(T_{2}, T_{1}\left[R_{1}\right]\right)$;

3) if $T_{1} \neq \varnothing$ and $T_{2}=\varnothing$, then $S=\operatorname{Del}\left(T_{1}\left[R_{1}\right], T_{1}\right)$;

4) if $T_{1} \neq \varnothing$ and $T_{2} \neq \varnothing$, then $R_{1} \equiv R_{2}$ and $S=S_{1} \ldots S_{h}$, where $S_{i}$ defines the transformation: $\mathrm{T}_{1}\left[(\mathrm{i}-\mathrm{th}) \operatorname{son}\left(\mathrm{R}_{1}\right)\right] \alpha_{\mathrm{Si}} \mathrm{T}_{2}\left[(\mathrm{i}-\mathrm{th}) \operatorname{son}\left(\mathrm{R}_{2}\right)\right], 1 \leq \mathrm{i} \leq \mathrm{h}$.

Note the transformation from $\mathrm{T}_{1}$ to $\mathrm{T}_{2}$ is uniquely determined. We can now pose:

Definition 1. For two ordered h-ary trees $T_{1}, T_{2}$, with $T_{1} \alpha_{S} T_{2}$, the subtree dinstance $d_{s}\left(T_{1}, T_{2}\right)$ is given by $|S|$.

For $h=2$, consider the binary trees $T_{1}, T_{2}$ of fig. 1 (external nodes are denoted by $\Delta$ ). The transformation sequence is the following:

$\mathrm{S}=\mathrm{Ins}\left(\mathrm{T}_{2}[3], \mathrm{T}_{1}[*]\right), \operatorname{Del}\left(\mathrm{T}_{1}[3], \mathrm{T}_{1}\right), \operatorname{Del}\left(\mathrm{T}_{1}[7], \mathrm{T}_{1}\right)$, Ins $\left(\mathrm{T}_{2}[6], \mathrm{T}_{1}[@]\right)$. 
We then have $d_{s}\left(T_{1}, T_{2}\right)=4$, while the node correspondences $1 \equiv 1,2 \equiv 2,5 \equiv 4,6 \equiv 5$ are established.
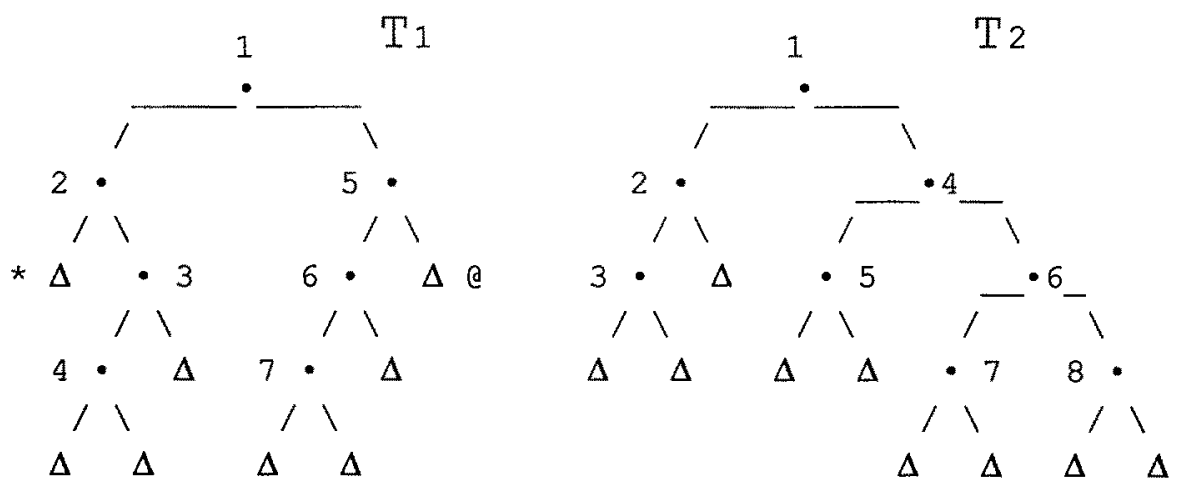

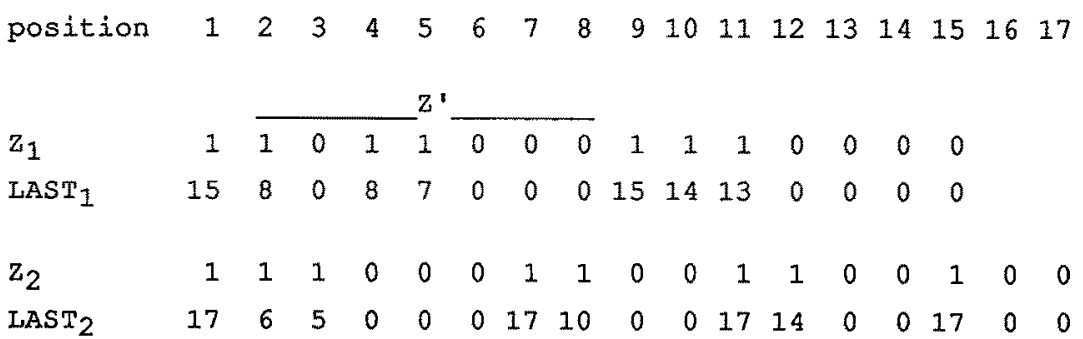

Fig. 1

An ordered h-ary tree $\mathrm{T}$ can be represented with a binary Zaks sequence $\mathrm{Z}$, obtained by labelling the internal nodes of $T$ by 1 , and the external nodes by 0 , and then visiting $T$ in preorder [7]. $Z$ has $n$ ones and $(h-1) n+1$ zeroes, and no prefix of $Z$ has $n^{\prime}$ ones and $(h-1) n^{\prime}+1$, $n^{\prime}<n$. Moreover, each subtree $T^{\prime}$ of $T$ corresponds to a subsequence $Z^{\prime}$ of $Z$, which is the Zaks sequence for $T^{\prime}$. See for example fig. 1 , where $Z_{1}$ and $Z_{2}$ are the $Z$ aks sequences for $T_{1}$ and $T_{2}$, respectively. The Zaks subsequence $Z$ ' of $Z_{1}$ corresponds to $T_{1}[2]$.

We now pose:

Problem 1. Given two ordered $h$-ary trees $P, T$, and an integer $k \geq 0$, find all the nodes $N \in T$ such that $d_{s}(P, T[N]) \leq k$.

For $\mathrm{k}=0$ and $\mathrm{h}=2$ this problem becomes the well known subtree isomorphism problem between ordered binary trees. For $\mathrm{k}>0$ we have an approximate tree matching problem. As noted in [9], such a problem can be seen as a generalization of the approximate string matching problem applied to trees.

We solve problem 1 using the Zaks representation of the trees, and a suffix tree built on it. 
Recall that, for a given sequence $X$ of $n$ elements, the suffix tree on $X$ allows to compute, in time $O(1)$, the lenght PREFIX $(X, i, j)$ of the longest common prefix between the subsequences $X(i), \ldots, X(n)$ and $X(j), \ldots, X(n)[3]$.

Let $Z(i)$ denote the $i$-th element of a Zaks $Z$, and $N_{i}$ be the tree node corresponding to $Z(i)$. Define the new sequence LAST, with $|L A S T|=|Z|$, as follows. For $Z(i)=0$ ( $N_{i}$ external), $\operatorname{LAST}(\mathrm{i})=0$. For $\mathrm{Z}(\mathrm{i})=1\left(\mathrm{~N}_{\mathrm{i}}\right.$ internal), $\operatorname{LAST}(\mathrm{i})=\mathrm{j}$, where $\mathrm{j}$ is the last position in $\mathrm{Z}$ of the subsequence $Z$ ' corresponding to the subtree rooted at $N_{i}$. For example, the sequences $\mathrm{LAST}_{1}$, LAST $_{2}$ for the trees $T_{1}, T_{2}$ are shown in fig.1. Similarly define the sequence PROX, with $|P R O X|=|Z|$, as follows. if $Z(i)=0$, then $\operatorname{PROX}(i)=0$; if $Z(i)=1$, then $\operatorname{PROX}(\mathrm{i})=j$, where $j>i$ is the minimum value for which $Z(j)=1$ (if $Z(j)=0$ for all $j>i$, then $\operatorname{PROX}(i)=|Z|+1$ ).

Problem 1 can be solved with the following method, consisting of a preprocessing phase followed by algorithm 1 :

\section{Preprocessing}

1. build the Zaks sequences $Z_{\mathrm{P}}, \mathrm{Z}_{\mathrm{T}}$ for $\mathrm{P}$ and $\mathrm{T}$;

2. build the LAST sequences $\mathrm{LAST}_{\mathrm{P}}, \mathrm{LAST}_{\mathrm{T}}$ for $\mathrm{P}$ and $\mathrm{T}$;

3 . build the sequence PROX for $T ;\{$ note: PROX contains pointers to the successive nonvoid subtrees, in preorder);

4. build the suffix tree for the string $X=Z_{T} \$ Z_{p} \&$ ( $\$, \&$ are markers).

\section{Algorithm 1}

$t:=1$

while $t \leq\left|Z_{T}\right|$ do

begin

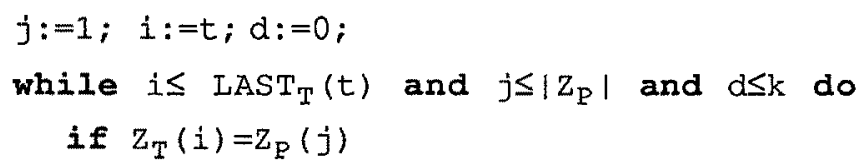

1: then begin $r:=\operatorname{PREFIX}\left(x, j, j+\left|z_{T}\right|+1\right)$;

$$
i:=i+r ; j:=j+r \text { end }
$$

2: else if $z_{T}(i)=0$ and $z_{P}(j)=1$

$$
\begin{aligned}
& \text { then begin } i:=i+1 ; j:=\operatorname{LAST}_{P}(j)+1 ; d:=d+1 \text { end } \\
& \text { else begin } i:=\operatorname{LAST}_{T}(i)+1 ; j:=j+1 ; d:=d+1 \text { end; }
\end{aligned}
$$

if $a \leq k$ then $\operatorname{PRINT}(t)$; we have $d_{S}(P, T[t]) \leq k$ \}

$t:=\operatorname{PROX}(t)$

end

end algorithm 1 .

Theorem 1. Problem 1 is solved in time $\mathrm{O}(\mathrm{h} \cdot|\mathrm{P}|+\max (\mathrm{h}, \mathrm{k}) \cdot \mid \mathrm{Tl}))$, using preprocessing and algorithm 1.

Proof. Correctness. Let $\mathrm{N}_{t}$ be the node of $\mathrm{T}$ corresponding to $\mathrm{Z}_{\mathrm{T}}(\mathrm{t})$. The inner while loop of algorithm 1 computes the distance between $\mathrm{P}$ and $\mathrm{T}\left[\mathrm{N}_{\ell}\right]$. The outer while loop iterates such 
a computation to all the nonvoid subtrees of $T$, pointed by the function PROX[t].

Complexity. Preprocessing. Steps 1 and 2 can be executed in time $\mathrm{O}(\mathrm{h}(\mathrm{IPI}+\mid \mathrm{TI})$ ) (size of the output from preprocessing), since $\mathrm{Z}_{\mathrm{P}}, \mathrm{Z}_{\mathrm{T}}, \mathrm{LAST}_{\mathrm{P}}$ and $\mathrm{LAST}_{\mathrm{T}}$ can be directly built visiting the two trees. Step 3 amounts to scanning $Z_{T}$, hence takes time $O(h / T)$. Step 4 takes time $\mathrm{O}(\mathrm{h}(|\mathrm{P}|+\mid \mathrm{TI}))$ [3]. The total time of preprocessing is therefore $\mathrm{O}(\mathrm{h}(|\mathrm{P}|+|\mathrm{T}|))$. To analyze algorithm 1, consider the inner while loop first. The else statement, labelled 2 , is executed at most $\mathrm{k}+1$ times (at this point the while is terminated). When a then statement, labelled 1 , is executed, the function PREFIX bring the indices $i, j$ to two values for which $Z_{T}(i) \neq Z_{P}(j)$. That is, at the next while iteration, the else statement is again executed. Therefore, the then statement is executed at most as many times as the else statement. Since PREFIX can be computed in $O(1)$, the total time required by the inner while loop is $O(k)$, for $k>0$. For $k=0$ the while loop is executed exactly once, with a single computation of PREFIX. Each iteration of the outer loop performs a comparison between $\mathrm{P}$ and a subtree of $\mathrm{T}$, and this comparison is repeated for all the internal nodes of $\mathrm{T}$. Therefore, the outer loop is executed $\mathrm{O}(\mathrm{ITI})$ times, and the overall time complexity of algorithm 1 is $O(k|T|)$ for $k>0$, or $O(|T|)$ for $k=0$.

If the internal nodes of the two trees are labelled, another distance may be considered in addition to $\mathrm{d}_{s}$. Let $\Sigma$ be a finite alphabet, $0 \notin \Sigma$, and let $\lambda(\mathrm{N}) \in \Sigma$ be the label of a node $\mathrm{N}$. Disregarding the labels, consider the transformation $T_{1} \alpha_{s} T_{2}$, and let $A_{1}, \ldots, A_{h} \in T_{1}$ and $B_{1}, \ldots, B_{h} \in T_{2}$ be the ordered sets of nodes put into correspondance in the trasformation. That is $A_{i} \equiv B_{i}, 1 \leq i \leq h$. (Note that $A_{i}$ and $B_{i}$ belong to the common portion of $T_{1}$ and $T_{2}$ ). We pose:

Definition 2. For two labelled ordered h-ary trees $\mathrm{T}_{1}, \mathrm{~T}_{2}$, the mismatch distance $\mathrm{d}_{\mathrm{m}}\left(\mathrm{T}_{1}\right.$, $\left.T_{2}\right)$ is given by the number of pairs $A_{i}, B_{i}$ such that $\lambda\left(A_{i}\right) \neq \lambda\left(B_{i}\right)$.

Problem 1'. Given two labelled ordered $h$-ary trees $P, T$, and two integers $k, k_{1} \geq 0$, find all the nodes $N \in T$ such that $d_{s}(P, T[N]) \leq k, d_{m}(P, T[N]) \leq k_{1}$.

Problem 1' can be solved with a variant of algorithm 1, using the labelled Zaks sequences $Z_{\lambda T}, Z_{\lambda P}$, that is, Zaks sequences with the 1's replaced by the internal node labels; and taking into account that $Z_{\lambda T}(i) \neq Z_{\lambda P}(j)$ may occur for a label mismatch. With an argument similar to the one used in theorem 1 , it can be easily shown that the inner while loop of the algorithm is executed at most $2\left(\max \left(k, k_{1}\right)+1\right)$ times. Therefore we have:

Corollary 1. Problem 1' can be solved in time $O\left(h \cdot|P|+\max \left(h, k_{1}, k_{2}\right) \cdot|T|\right)$.

\section{Matching arbitrary ordered trees}

If the node degree is arbitrary, the transformation of a tree into another by inserting and deleting subtrees becomes less obvious. For a nonvoid ordered tree $\mathrm{T}$, and a node

$\mathrm{N} \in \mathrm{T}$, we newly define these operations as follows:

1) for $N \in T, N$ leaf, insert a non void tree $T_{1}$ in $T$ in the place of $N$. Denoted by: Ins $\left(T_{1}, T[N]\right)$; 
2) for $N \in T, N$ non leaf, delete $T[N]$ from $T$ by replacing $T[N]$ with the single node $N(N$ becomes a leaf). Denoted by $\operatorname{Del}(\mathrm{T}[\mathrm{N}], \mathrm{T})$.

Note that we do not use now the concept of extenal and internal nodes. Let a sequence $S$ of insert and delete operations determine the transformation of a tree $T_{1}$ into another tree $T_{2}$, denoted by $T_{1} \beta_{S} T_{2}$. Letting $R_{1}, R_{2}$ be the roots of $T_{1}, T_{2}$, respectively, $S$ is such that:

1) $R_{1} \equiv R_{2}$,

2) if $\left|T_{1}\right|=\left|T_{2}\right|=1$ then $S=\varnothing$;

3) if $\left|T_{1}\right|=1$ and $\left|T_{2}\right|>1$, then $S=\operatorname{Ins}\left(T_{2}, T_{1}\left[R_{1}\right]\right)$;

4) if $\left|T_{1}\right|>1$ and $\left|T_{2}\right|=1$, then $S=\operatorname{Del}\left(T_{1}\left[R_{1}\right], T_{1}\right)$;

5) if $\left|T_{1}\right|>1$ and $\left|T_{2}\right|>1$, and $\operatorname{deg} R_{1}=\operatorname{deg} R_{2}=h$, then $S=S_{1} \ldots S_{h}$, where $S_{i}$ defines the transformation: $T_{1}\left[(i-t h) \operatorname{son}\left(R_{1}\right)\right] \beta_{S i} T_{2}\left[(i-t h) \operatorname{son}\left(R_{2}\right)\right], 1 \leq i \leq h$.

$S$ is undefined if $\left|T_{1}\right|>1,\left|T_{2}\right|>1$, and deg $R_{1} \neq \operatorname{deg} R_{2}$; or $\left|T_{1}\right|>1,\left|T_{2}\right|>1$, and deg $R_{1}=\operatorname{deg}$ $R_{2}$, but one of the sequences $S_{i}$ of point 5 is undefined. In this case $\beta_{S}$ does not exist, that is, $\mathrm{T}_{1}$ cannot be transformed into $\mathrm{T}_{2}$ by insert or delete operations. If $S$ is defined, the transformation is uniquely determined. We now pose:

Definition 3. For two ordered trees $T_{1}, T_{2}$, with $T_{1} \beta_{S} T_{2}$, the leaf distance $d_{1}\left(T_{1}, T_{2}\right)$ is given by ISI.

In the sample trees $T, P$ of fig. 2 we have $d_{l}(T, P)=2, d_{l}(T[2], P)=0$, while $d_{1}(T[1], P)$ is undefined.
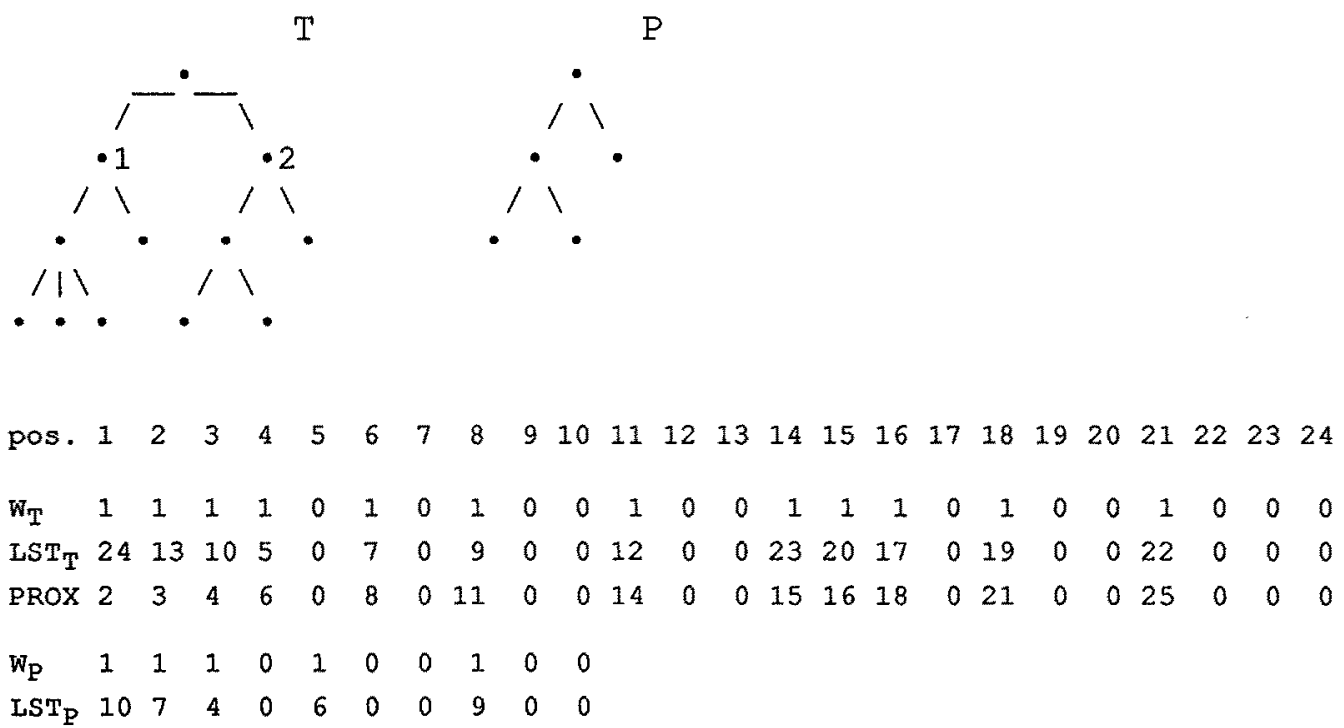
An ordered tree $\mathrm{T}$ can be represented by a binary sequence $W$, obtained visting $\mathrm{T}$ in preorder. $\mathrm{A} 1$ is entered in $\mathrm{W}$ for each node encountered in the visit, and $\mathrm{a} 0$ is entered for each return to the previous level. The syntactic stucture of the sequence $\mathrm{W}$ is the following:

$\mathrm{W}::=1 \mathrm{~W}^{*} 0$

where a term $\mathrm{W}$ occurs for each subtree of the root. In fact, each subtree $\mathrm{T}^{\mathrm{t}}$ of $\mathrm{T}$ corresponds to a subsequence $\mathrm{W}^{\prime}$ of $\mathrm{W}$. Clearly $\mathrm{W}$ has the following properties:

1. W has ITI ones and IT| zeroes;

2. all prefixes of $W$ have a number of ones greater than the number of zeroes.

For example, see the sequences $\mathrm{W}_{\mathrm{T}}, \mathrm{W}_{\mathrm{P}}$ for the trees $\mathrm{T}$ and $\mathrm{P}$ reported in fig.2. An easy inductive argument shows that here is a one to one correspondence between ordered trees and sequences W. We denote by W(i) the $\mathrm{i}$-th position of $\mathrm{W}$, and define the sequence LAST as for problem 1 (see fig.2).

We then pose:

Problem 2. Given two ordered trees $P, T$, and an integer $k \geq 0$, find all the nodes $N \in T$ such that $d_{1}(P, T[N]) \leq k$.

If we compute $d_{1}$ excluding case 4 from the definition of $S$ (that is we consider $S$ to be undefined for $|\mathrm{P}|>1$ and $|\mathrm{T}|=1$ ), and take $\mathrm{k}=|\mathrm{P}|$, then problem 2 becomes the tree pattern matching defined in [5].

Problem 2 is solved with the preprocessing phase and algorithm 2 reported below (refer to the example of fig.2). We have:

Theorem 2. Problem 2 is solved in time $\mathrm{O}(|\mathrm{P}|+\mathrm{k} \cdot|\mathrm{T}|))$, using preprocessing and algorithm 2 . Proof. Correctness. The inner while loop of algorithm 2 cheks the matchings of $P$ with a subtree of $T$. A point of mismatch, e. $g . W_{T}(i)=1, W_{P}(j)=0$, is reached via a PREFIX or a LAST computation. In both cases, $W_{T}(i-1)=W_{P}(j-1)$. If these carachters are 0 , we are attempting to matching internal nodes of $\mathrm{P}$ and $\mathrm{T}$ of different degrees. Hence $\mathrm{P}$ is not found (defined=false). If $W_{T}(i-1)=W_{P}(j-1)=1$, then $W_{T}(i-1)$ corresponds to an internal node $N$ of $T$ and $W_{P}(j-1)$ corresponds to a leaf $L$ of $P$. Then, the process goes on matching the subtree routed in $N$ with the leaf $L$, and increasing $d$. The case $W_{T}(i)=0, W_{P}(j)=1$ is symmetrical. The outer while loop iterates the procedure, matching $\mathrm{P}$ with to all the nonvoid subtrees of $\mathrm{T}$.

Complexity. Preprocessing takes time $\mathrm{O}(\mathrm{IP}|+| \mathrm{T} \mid)$ (see the proof of theorem 1). The inner while loop of algorithm 2 is executed at most $2 k+1$ times. In fact, $\leq k+1$ times for the common subsequences starting at $\mathrm{W}_{\mathrm{T}}(\mathrm{i})=\mathrm{W}_{\mathrm{P}}(\mathrm{j})$, interleaved with the determinations of $\leq \mathrm{k}$ mismatches between a leaf and a subtree. If a forbidden mismatch occurs (defined=true) the loop is forcibly terminated. Each iteration of the outer while loop performs a comparison between $\mathrm{P}$ and a subtree of $\mathrm{T}$, hence this loop is executed $\mathrm{O}(\mathrm{TT} \mid$ times. The complexity of algorithm 2 is then $O(k \mid T I)$.

We can consider labelled ordered trees, extend the definition of mismatch distance $d_{m}$ (Definition 2) to this case, and apply an obvious extension of algorithm 2. We have:

Problem 2'. Given two labelled ordered $P, T$, and two integers $k, k_{1} \geq 0$, find all the nodes $\mathrm{N} \in \mathrm{T}$ such that $\mathrm{d}_{1}(\mathrm{P}, \mathrm{T}[\mathrm{N}]) \leq \mathrm{k}, \mathrm{d}_{\mathrm{m}}(\mathrm{P}, \mathrm{T}[\mathrm{N}]) \leq \mathrm{k}_{1}$.

Corollary 2. Problem $2 '$ can be solved in time $\mathrm{O}\left(\left(\mathrm{P}\left|+\max \left(\mathrm{k}, \mathrm{k}_{1}\right) \cdot\right| \mathrm{T} \mid\right)\right.$. 


\section{Preprocessing}

1. build the sequences $W_{P}, W_{T}, \operatorname{LAST}_{\mathrm{P}}, \mathrm{LAST}_{\mathrm{T}}$ for $\mathrm{P}$ and $\mathrm{T}$ and the sequence PROX for T;

2. build the suffix tree for the string $X=W_{T} \$ W_{P} \&$ ( $\$, \&$ are markers).

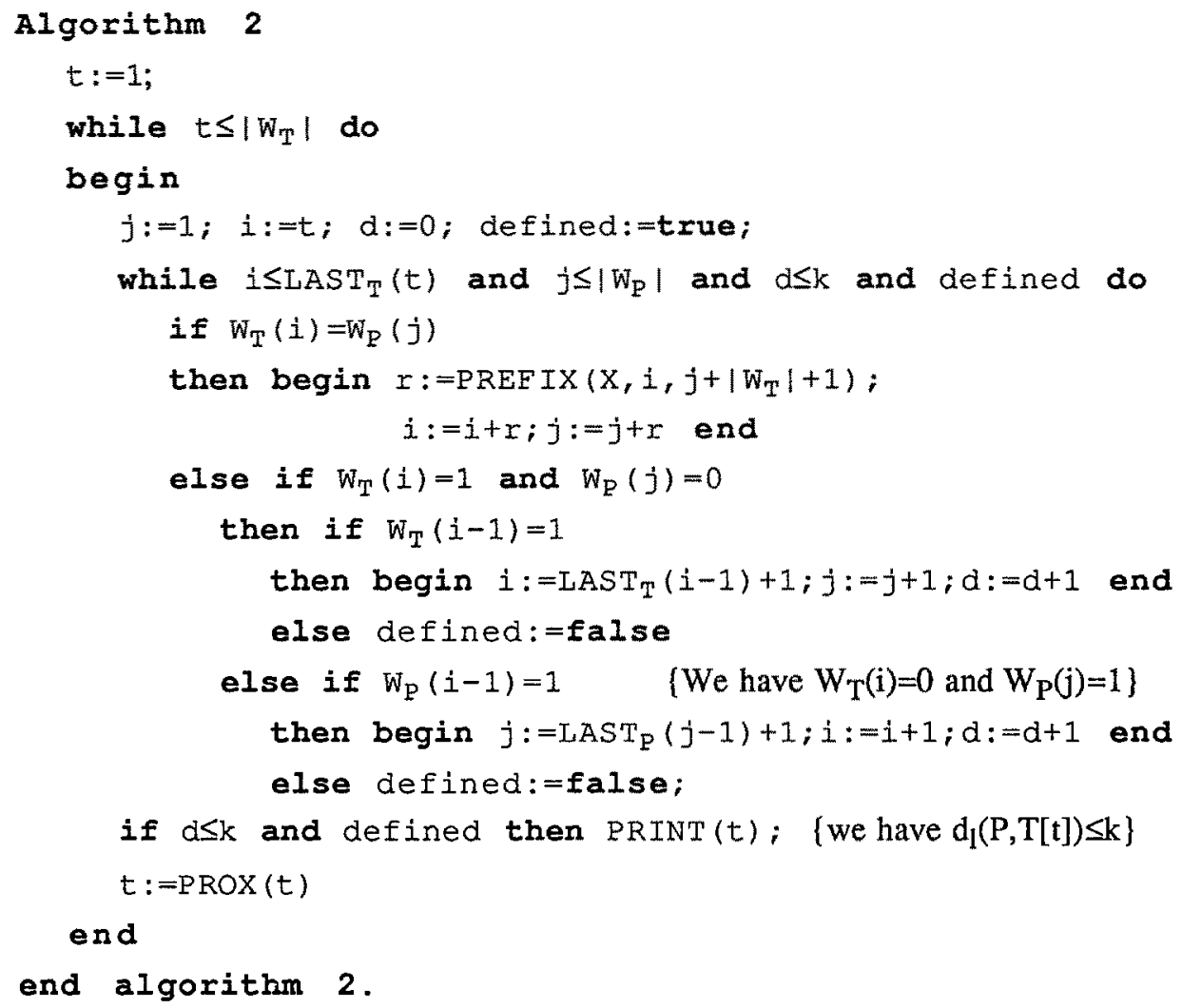

\section{Acknowledgment.}

This work has been partially supported by M.U.R.S.T. of Italy.

\section{References}

[1] M. Dubiner, Z. Galil and E. Magen, Faster tree pattern matching, Proc. 31 -st IEEE Symp. on Found. of Comp. Sc.(1990) 145-149.

[2] Z. Galil and R. Giancarlo, Data structures and algorithms for approximate string matching, J. Complexity 4 (1988) 33-72.

[3] D. Harel and R.E. Tarjan, Fast algorithms for finding nearest common ancestors, SIAM J. Comp.13 (1984) 338-355.

[4] C.M. Hoffmann and M.J. O'Donnell, Pattern matching in trees, J. ACM 29 (1982) 68-95. 
[5] S.R. Kosaraju, Efficient tree pattern matching, Proc. 30-th IEEE Symp. on found. of Comp. Sc.(1989) 178-183.

[6] G.M. Landau and U. Vishkin, Introducing efficient parallelism into approximate string matching and a new serial algorithm, Proc. 18-th ACM Symp. on Theory of Comp. (1986) 220-230.

[7] E. Mäkinen, On the subtree isomorphism problem for ordered trees, Inf. Proc. Let. 32 (1989) 271-273.

[8] L. Stryer, Biochemestry, 3-rd edition, W.H.Freeman and Co., New York, NY 1988.

[8] K. Zhang and D. Shasha, Simple fast algorithms for the editing distance between trees and related problems, SIAM J. Comp. 18 (1989) 1245-1262. 